\title{
Using Heuristics to Speed up Induction on Continuous-Valued Attributes
}

\author{
Günter Seidelmann \\ Hahn-Meitner-Institut Berlin GmbH \\ Bereich Datenverarbeitung und Elektronik \\ Glienicker Str. 100, W-1000 Berlin 39, Germany \\ Email: seidelmann@vax.hmi.dbp.de
}

\begin{abstract}
Induction of decision trees in domains with continuous-valued attributes is computationally expensive due to the evaluation of every possible test on these attributes. As the number of tests to be considered grows linearly with the number of examples, this poses a problem for induction on large databases. Two variants of a heuristic, based on the possible difference of the entropy-minimization selection-criterion between two tests, are proposed and compared to a previously known heuristic. Empirical results with real-world data confirm that the heuristics can reduce the computational effort significantly without any change in the induced decision trees.
\end{abstract}

\section{Introduction}

Learning from examples has been recognized as a promising way of acquiring knowledge for classification purposes. Especially top-down induction of decision trees (TDIDT) has been used successfully in a number of applications (see e. $\mathrm{g}$. [2], [3], [5]).

Early TDIDT-methods are restricted to nominal attributes. But in many applications, e.g. in process control, which include sensory data, continuous-valued attributes are predominant and should be treated adequately. A straightforward extension, as briefly mentioned in [4], leads to good results, but has the disadvantage of a rather high computational effort, which increases with the number of examples. On large data bases the runtime of the induction can therefore become inacceptable. This aspect has not found much attention until Fayyad and Irani [1] recently proposed a heuristic to reduce the average complexity while preserving the induced trees.

This paper describes two versions of a supplementary heuristic and argues that a combination with the one developed by Fayyad and Irani can reduce the computational effort significantly. The characteristics of the heuristics are discussed and their payoff is tested on different sets of real-world data.

Sect. 2 briefly describes the ID3 algorithm and the results of Fayyad and Irani. In Sect. 3 another approach is motivated and two variants of a new heuristic are given. The results of empirical comparisons are presented in Sect. 4. Finally, conclusions can be found in Sect. 5 . 


\section{Induction on Continuous-Valued Attributes}

Induction of decision trees typically expects the examples to be represented as a set of attribute-value pairs with respect to a common set of attributes with each example belonging to one of a disjoint set of classes. In general, the algorithms select a test on one or more of the attributes to partition the set of examples into subsets, which are further divided recursively until a termination criterion is met. The generated tree consists of the selected tests at the inner nodes with subtrees for each possible outcome. The leaves are marked with the predominant class of the corresponding subset.

The well-known ID3 [3] selects the test which minimizes the average entropy $E(T)$ in the resulting subsets. Let $C_{1}, \ldots, C_{k}$ be the classes occuring in the example set, $P\left(C_{i}\right)$ the corresponding probabilities, $T$ a test on the examples which partitions the set into subsets $T_{1}, \ldots, T_{m}, P\left(T_{j}\right)$ the probability of an example to fall into the subset $T_{j}$ and let $P\left(C_{i} \mid T_{j}\right)$ denote the conditional probability of an example out of $T_{j}$ belonging to class $C_{i}$. The remaining average entropy $E(T)$ after the evaluation of a test $\mathrm{T}$ is then given by:

$$
E(T)=-\sum_{j=1}^{m} \sum_{i=1}^{k} P\left(T_{j}\right) P\left(C_{i} \mid T_{j}\right) \log _{2}\left(P\left(C_{i} \mid T_{j}\right)\right)
$$

For continuous-valued attributes tests of the form $A(x)<V$, with $A(x)$ denoting the attribute value of an example $x$, are generated by varying the threshold value $\mathrm{V}$. This can be done efficiently by ordering the examples according to their attribute values and representing the tests as a splitting of this list (see Fig. 1). This admits an incremental computation of the probabilities needed in (1).

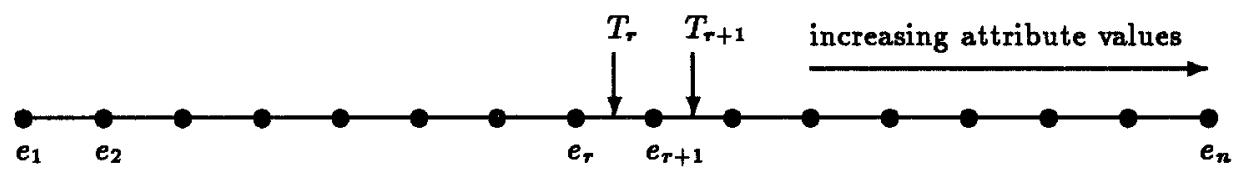

Fig. 1. Tests splitting the ordered list of examples

The number of possible tests $A(x)<V$ is linear in the number of examples ${ }^{1}$, whereas only one test needs to be evaluated for a nominal attribute. Therefore the runtime of TDIDT- algorithms grows significantly in the presence of continuous-valued attributes, so that induction on large data bases becomes a time-consuming effort.

Not every possible test needs to be evaluated, however. In the ordered list of examples (see Fig. 1) two adjacent examples $e_{r}$ and $e_{r+1}$ often belong to

\footnotetext{
${ }^{1}$ If different examples have the same attribute value, the number of tests is, of course, sublinear.
} 
the same class. Intuitively, it does not seem to make sense to divide the set of examples between them. Fayyad and Irani prove that in terms of the entropyminimization selection criterion used in ID3 either the division before example $e_{r}$ or after example $e_{r+1}$ is indeed superior to the one between them. They introduce the term boundary point for those thresholds whose constituting examples $e_{r}$ and $e_{r+1}$ belong to different classes.

As tests between boundary points are always inferior to at least one of their neighbouring tests, the following heuristic can be used:

Heuristic 1. In the test selection process only those tests need to be evaluated which fall on boundary points.

The achievable reduction in the number of test evaluations depends heavily on the data. Given $n$ examples out of $k$ different classes, a perfectly predicting attribute yields only $k-1$ boundary points, whereas a totally uncorrelated attribute can be expected to result in $(n-1) \frac{k-1}{k}$ boundary points. This indicates that Heuristic 1 will work particularly well on data sets which contain few classes.

\section{Heuristics Based on the Maximum Difference}

In order to select a test at a node during the induction, the algorithm has to generate and evaluate the possible tests on all available attributes. The test yielding the minimum remaining entropy (1) is selected. If there are many examples and a continuous-valued attribute is considered, two successive tests $T_{r}$ and $T_{r+1}$ (see Fig. 1) have a very small difference in their rating by the selection criterion. With an estimation of this difference the evaluation of test $T_{\tau+1}$ can safely be omitted if test $T_{\boldsymbol{r}}$ is sufficiently inferior to a previously found one.

Hence an upper bound is needed for the difference between $E\left(T_{r+1}\right)$ and $E\left(T_{r}\right)$ with the two tests differing only in the example $e_{r+1}$. Such a bound is given in Theorem 1.

Theorem 1. Given $n$ examples $e_{1}, \ldots, e_{r}, e_{r+1}, \ldots, e_{n}$ and two successive tests $T_{r}$ and $T_{r+1}$ which split the examples after $e_{r}$ and $e_{r+1}$ (resp.) the following holds:

$$
E\left(T_{r}\right)-E\left(T_{r+1}\right) \leq \frac{2 \log _{2}(n+1)-\log _{2} r}{n}=\max -\operatorname{diff}\left(T_{r}\right)
$$

Let now $E_{\min }$ be the minimum remaining entropy reached so far by a test during the test selection. If a test $T_{r}$ results in an entropy $E\left(T_{r}\right)$ such that $E\left(T_{r}\right)$ - max-diff $\left(T_{r}\right)>E_{m i n}$, the following test $T_{r+1}$ does not have to be considered. Furthermore, as the function max-diff( $\left(T_{r}\right)$ decreases strictly monotonously in $\mathrm{r}$, even more tests can eventually be eliminated. This leads to:

Heuristic 2. If the minimal remaining entropy reached so far is given by $E_{m i n}$, the next test to be evaluated after test $T_{r}$ is $T_{r+\Delta}$ with:

$$
\Delta=\max \left(1,\left\lceil\frac{E\left(T_{r}\right)-E_{\min }}{\max -\operatorname{diff}\left(T_{r}\right)}\right\rceil\right)
$$


On close examination the heuristic shows the following properties:

- As $\Delta$ depends on $E_{\min }$, the number of tests to be omitted is influenced by the order in which the attributes are processed. To achieve high savings the most relevant attribute should be chosen first.

- Since max-diff $\left(T_{r}\right)$ is monotonously decreasing in $r$, the $\Delta$-values are higher in the second half of the list.

- In general, Heuristic 2 will be more effective on irrelevant attributes than on relevant ones, provided a relevant attribute has been processed previously.

The following extension takes advantage of some of these properties. As higher benefits are obtained in the second half of the lists, it can be useful to start with a test in the middle of the list of examples and to proceed successively to the borders, using Heuristic 2.

Now the first test to be evaluated for an attribute is the one which splits the set of examples in the middle. These tests will have different entropy measures depending on the class distribution in the two subsets. Their entropy measures can be used to estimate the relevance of the attributes. By processing those attributes first that yield low entropy measures, an optimal order of the attributes can be approximated. This method is stated in the following heuristic:

Heuristic 3. For each attribute $A_{i}$ the examples are partitioned at the median into two roughly equally large sets by a test $T_{m_{i}}$. The examples in the set with attribute values below or above the median are ordered by decreasing or increasing attribute values resp. These lists are ordered by increasing values of $E\left(T_{m_{i}}\right)$ and processed according to Heuristic 2.

\section{An Empirical Comparison}

As the efficiency of the heuristics discussed depends heavily on the characteristics of the data, an evaluation using real-world data is needed. In these experiments the heuristics are used on their own as well as in combinations.

The data sets used are listed in Table 1 together with a brief description. Except for the solar data all of them are publicly available; as far as training and test sets are supplied separately, they were joined for the following experiments. Examples with values missing were removed.

As the heuristics aim for a reduction in the number of tests to be evaluated by the entropy-minimization selection-criterion, the number of tests actually considered during the induction gives a first metric for comparison. It is a machineand implementation-independent efficiency measure of the heuristics, which ignores the overhead associated with them. The results for different data sets are given in Fig. 2.

Some observations should be discussed:

- The heuristics based on the max-diff bound perform poorly on the medical domains cleve1, cleve2 and pima. These domains contain many examples with identical attribute values, thus reducing the number of possible tests that are to be omitted despite high $\Delta$-values. 
Table 1. Brief description of the data sets

\begin{tabular}{|l|r|r|r|r|}
\hline Domain & Name & Examples & Attributes & Classes \\
\hline Heart-diseases & cleve1 & 297 & 13 & 2 \\
Same as above, but 5 classes & cleve2 & 297 & 13 & 5 \\
Glass types & glass & 214 & 9 & 6 \\
Diabetes & pima & 768 & 8 & 2 \\
Semiconductor films & solar & 540 & 10 & 4 \\
Sonar signals & sonar & 208 & 60 & 2 \\
Vowel recognition & vowel & 990 & 10 & 11 \\
\hline
\end{tabular}

- Heuristic 1 is more efficient on data sets with few classes. It yields exceptionally high reductions on the sonar data and performs better on the cleve1 data with 2 classes than on the corresponding set cleve 2 with 5 classes.

- Only on the vowel data the more sophisticated Heuristic 3 performs considerably better than Heuristic 2, where obviously a more suitable order of attributes is found.

- The combinations of two heuristics consistently achieve good results. They reduce the number of test evaluations by at least $43 \%$ on the cleve 2 data up to $83 \%$ on the vowel data.

Reductions in the number of test evaluations are achieved at the expense of the computational overhead associated with each heuristic. From a practical point of view the only interesting metric is reduction in total runtime. As expected, no substantial reductions are attained in the medical domains $c l e v e 1^{2}$, cleve2 and pima. However, in most cases the overhead of the heuristics is compensated by reductions in the number of test evaluations; only the large overhead of Heuristic 3 did not pay off.

Especially the combinations work reliably on the other data sets. The combination of Heuristic 1 with Heuristic 2 results in a speed-up between 20\% (sonar data) and $30 \%$ (vowel data); the combination with Heuristic 3 saves between $10 \%$ (glass data) and $39 \%$ (vowel data).

\section{Conclusion}

When decision trees are induced by means of the entropy-minimization selectioncriterion, the number of tests to be evaluated can be reduced by using simple heuristics. This result has been confirmed by empirical experiments. As the heuristics exploit different characteristics of the data, a combination of them will work reliably for a wide variety of applications.

The heuristics apply first of all to the entropy-minimization selection-criterion. In [1] Fayyad and Irani mention that their heuristic (Heuristic 1 in this paper) can be used with other selection measures as well. It should be possible to derive

${ }^{2}$ In [1] Fayyad and Irani report a runtime reduction of more than $15 \%$ for their heuristic on this data set. 


\begin{tabular}{|c|c|c|c|}
\hline & without heuristic & $\because=$ & Heuristic 1 \\
\hline$\because$ & Heuristic 3 & 00 & Heuristics $1 \& 2$ \\
\hline
\end{tabular}
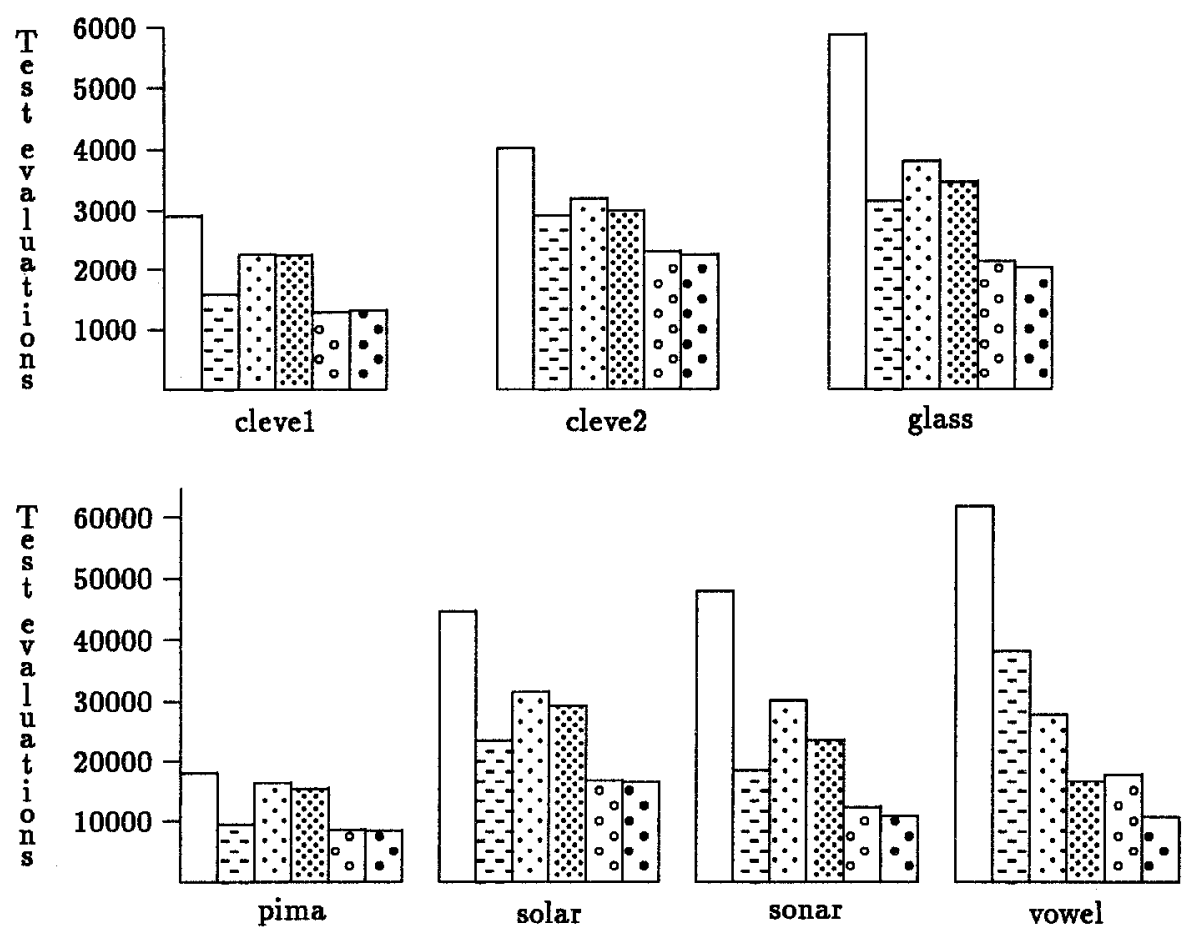

Fig. 2. Number of tests evaluated

bounds - similar to the one found for the entropy measure - for other selection criteria, so that Heuristics 2 and 3 could be applied to them as well.

\section{References}

1. U. M. Fayyad and K. B. Irani. On the handling of continuous-valued attributes in decision tree generation. Machine Learning, 8(1):87-102, 1992.

2. W. J. Leech. A rule-based process control method with feedback. ISA Transactions, 26(2):73-80, 1987.

3. J. R. Quinlan. Learning efficient classification procedures and their application to chess end games. In Machine Learning: An Artificial Intelligence Approach, pages 463-482. Tioga Press, 1983.

4. J. R. Quinlan. Simplifying decision trees. International Journal on Man-Machine Studies, 27:221-234, 1987.

5. J. C. Schlimmer. Learning meta knowledge for database checking. In Proceedings of the Ninth National Conference on Artificial Intelligence, pages 335-340. AAAI Press / The MIT Press, 1991. 